\title{
Nickel-catalyzed decarbonylative silylation, boronation and amination of arylamides via a deamidative reaction pathway
}

\author{
Shao-Chi Lee \\ Lin Guo ${ }^{a}$ \\ Huifeng Yue ${ }^{a}$ \\ Hsuan-Hung Liao ${ }^{a}$ \\ Magnus Rueping*a,b \\ a Institute of Organic Chemistry, RWTH Aachen University, \\ Landoltweg 1, D-52074 Aachen, Germany. E-mail: \\ Magnus.Rueping@rwth-aachen.de \\ ${ }^{\mathrm{b}}$ King Abdullah University of Science and Technology (KAUST), \\ KAUST Catalysis Center (KCC), Thuwal, 23955-6900 (Saudi \\ Arabia), E-mail: magnus.rueping@Kaust.edu.sa
}

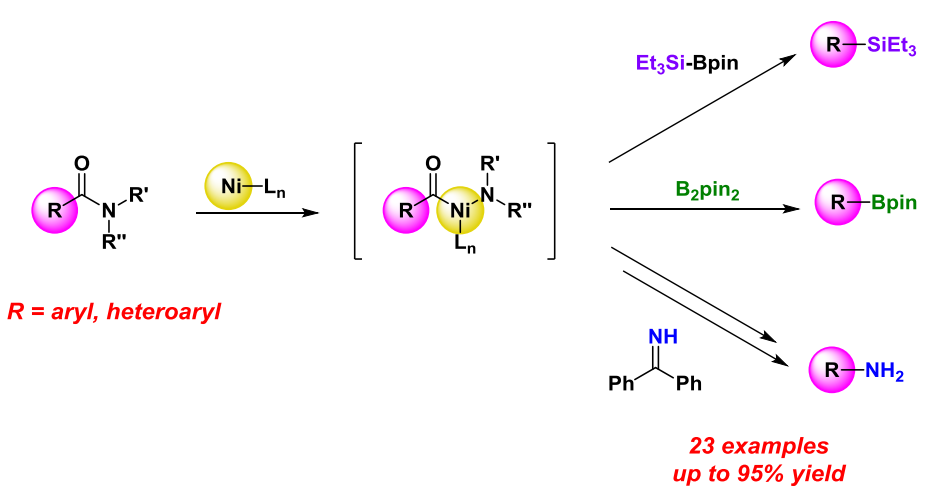

\begin{abstract}
Received:
Accepted:

Published online:

Abstract A nickel catalyzed decarbonylative silylation, boronation and amination of amides has been developed. This new methodology allows the direct interconversion of amides to arylsilanes, arylboronates and arylamines and enables a facile route for carbon-heteroatom bond formations in a straightforward and mild fashion.
\end{abstract}

Key words nickel, amides, arylsilanes, arylboronates and arylamines

Nickel-catalyzed cross-coupling reactions have become promising alternatives to traditional noble metal mediated reactions mainly due to the low-cost of nickel catalysts and the environmental friendly nature of coupling partners. ${ }^{1}$ Over the last 10 years, nickel has been widely used for cross-coupling reactions ${ }^{1,2}$ as well as for $\mathrm{C}-\mathrm{H}$ functionalizations. ${ }^{3}$ Initially, crosscoupling reactions were used to construct biaryls from halides and organometallic reagents. However, organohalides can be toxic compounds and wastes production is observed. Yet, due to the outstanding ability of oxidative-addition of nickel, ${ }^{1 \mathrm{~b}}$ a wide range of reactions can be performed with halide-free substrates by activating stable $\mathrm{C}-\mathrm{X}$ bonds, including $\mathrm{C}-\mathrm{S},{ }^{4} \mathrm{C}-\mathrm{O}^{5}$ and $\mathrm{C}-\mathrm{N}^{6}$ bonds. Nevertheless, the types of reactions disclosed are still limited, and hence new reactivities and reaction types are desirable.

In recent years, various decarbonylative cross-coupling reactions via transition metal-catalysis have been described. ${ }^{7}$ As conventional electrophilic substrates, acyl chlorides, ${ }^{8}$ acid anhydrides ${ }^{9}$ and esters ${ }^{10}$ are normally selected and used in decarbonylative reactions. In contrast, the amide $\mathrm{C}-\mathrm{N}$ bond cleavage is more challenging and requires particular attention. ${ }^{11}$ Amides exist abundantly in nature, e.g. in proteins and peptides and are widely used in artificial compounds like drugs and polymers. From the perspective of synthesis, amides would be ideal starting materials for their conversion to further functional groups. However, the $\mathrm{C}-\mathrm{N}$ bonds are highly stable and difficult to insert and cleave with transition metals. Therefore, direct methods for the transfer of amide moieties to other functional groups would be very advantageous and are highly demanded. ${ }^{12}$

Given our recent developments in the field of Ni-catalysis ${ }^{13}$ and in particular decarbonylative cross-coupling reactions with esters, we decided to expand our newly developed protocols to valuable amide substrates.

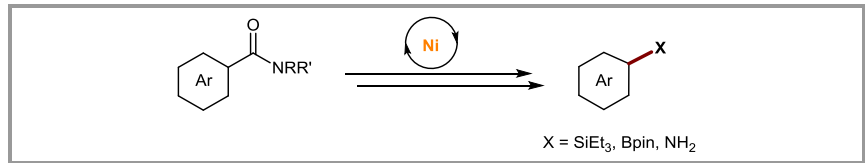

Scheme 1 New Ni-catalyzed decarbonylative cross-coupling reactions with amides.

Over the past few decades, organosilicon and organoboron compounds proved to provide a good platform for further functional group interconversion to access complex molecular scaffolds. ${ }^{14,15}$ Furthermore, their application in the synthesis of natural products and drug molecules is widely acknowledged. ${ }^{16}$, 17 In addition to organosilicon and organoboron derivatives, amines are further valuable intermediates in organic synthesis. ${ }^{18}$ Conventionally, organosilicon and organoboron compound are synthesized from sensitive Grignard reagents or organolithium reagents. ${ }^{19}$ Recently, transition-metal catalyzed silyl/boryl substitution of aryl halides ${ }^{20}$ and arene C-H silylation/borylation ${ }^{21}$ have been achieved. However, poor functional group tolerance, the utilization of expensive substrates, or site selectivity might cause limitations of the above methods. Herein, we report a practical method to achieve the silylation, borylation and amination of amides in an easy manner via $\mathrm{C}-\mathrm{N}$ bond cleavage. 
Recently, we and the Shi group have described the nickel/copper catalyzed decarbonylative silylation reaction of esters by applying silylboranes as coupling partners. ${ }^{100-p}$ In addition, Martin group described the nickel/copper catalyzed silylation of pivalates with silylboranes as coupling partners. ${ }^{22}$ Building up on these results, we started our investigation for the decarbonylative silylation of amides by examining various amides 1a-6a in reaction with silylborane $\mathbf{7}$ (Table 1). Firstly, we evaluated N-Boc, N-Benzyl and N-Boc, N-Phenyl imides 1a and $\mathbf{2 a}$, however only low yields of the desired arylsilane product 8a were observed (entries 1 and 2). Replacing the Boc protecting group with methyl and trifluoroacetyl groups (3a 4a) resulted in considerably lower yields (entries 3 and 4). Next, we turned our attention to sterically distorted amides $\mathbf{5 a}$ and $\mathbf{6 a}^{11}$ and were pleased to see that derivative $\mathbf{6 a}$ provided the desired product in good yield (entry 6). To further enhance the yield, we examined different reaction conditions. Use of 30 mol\% of $\mathrm{LiCl}$ as additive under the standard conditions did not improve the yield (entry 7). The effect of concentration was also evaluated, however no positive effect was observed upon increasing or decreasing the concentration (entries 8 and 9 vs 6). The yield slightly decreased when changing the solvent to 1,4-dioxane, which has a similar solubility and boiling point as toluene (entry 10).

Table 1 Optimization of arylsilane synthesis through decarbonylation of amides $^{a}$

\begin{tabular}{|c|c|c|c|c|c|}
\hline & 1a-6a & $\begin{array}{c}+\mathrm{E}_{3} \mathrm{Si}-\mathrm{Bpin} \\
7\end{array}$ & \multicolumn{2}{|c|}{$\begin{array}{l}\begin{array}{c}\mathrm{Ni}\left(\mathrm{COD}_{2}(10 \mathrm{~mol} \%)\right. \\
\mathrm{P}^{n} \mathrm{Bu}_{2}(40 \mathrm{~mol} \%) \\
\mathrm{CuF}_{2}(30 \mathrm{~mol} \%)\end{array} \\
\text { base }(3.0 \text { equiv. }) \\
\text { toluene, } 160{ }^{\circ} \mathrm{C}, 36 \mathrm{~h}\end{array}$} & $8 a$ \\
\hline Entry & $1 a-6 a$ & $\begin{array}{c}\mathrm{Ni}(\mathrm{COD})_{2} \\
(\mathrm{~mol} \%)\end{array}$ & Base & $\begin{array}{c}\text { Solvent } \\
\text { (conc., M) }\end{array}$ & $\begin{array}{l}\text { Yield } \\
(\%)^{b}\end{array}$ \\
\hline 1 & $1 a$ & 10 & $\mathrm{KF}$ & Toluene (0.2) & 9 \\
\hline 2 & $2 a$ & 10 & $\mathrm{KF}$ & Toluene (0.2) & 34 \\
\hline 3 & $3 a$ & 10 & $\mathrm{KF}$ & Toluene (0.2) & $<5$ \\
\hline 4 & $4 a$ & 10 & $\mathrm{KF}$ & Toluene $(0.2)$ & $<5$ \\
\hline 5 & $5 a$ & 10 & $\mathrm{KF}$ & Toluene (0.2) & 40 \\
\hline 6 & $6 a$ & 10 & $\mathrm{KF}$ & Toluene (0.2) & $83(79)^{c}$ \\
\hline $7^{d}$ & $6 a$ & 10 & $\mathrm{KF}$ & Toluene $(0.2)$ & 62 \\
\hline 8 & $6 a$ & 10 & $\mathrm{KF}$ & Toluene (0.4) & 68 \\
\hline 9 & $6 a$ & 10 & $\mathrm{KF}$ & Toluene $(0.13)$ & 34 \\
\hline 10 & $6 a$ & 10 & $\mathrm{KF}$ & Dioxane (0.2) & 61 \\
\hline 11 & $6 a$ & 5 & $\mathrm{KF}$ & Toluene (0.2) & 50 \\
\hline 12 & $6 a$ & 10 & CsF & Toluene $(0.2)$ & 30 \\
\hline 13 & $6 a$ & 10 & $\mathrm{Cs}_{2} \mathrm{CO}_{3}$ & Toluene $(0.2)$ & 36 \\
\hline 14 & $6 a$ & 10 & $\mathrm{~K}_{3} \mathrm{PO}_{4}$ & Toluene (0.2) & 41 \\
\hline $15^{\mathrm{e}}$ & $6 a$ & 10 & $\mathrm{KF}$ & Toluene $(0.2)$ & 0 \\
\hline
\end{tabular}

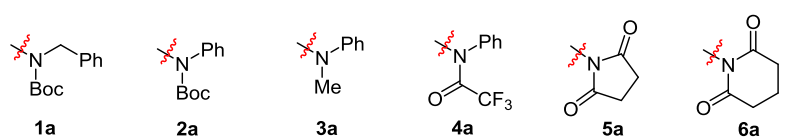

Reaction conditions: 1a-6a $(0.20 \mathrm{mmol}), 7$ (96.6 mg, $0.40 \mathrm{mmol}), \mathrm{Ni}(\mathrm{COD})_{2}(5.5$ $\mathrm{mg}, 10 \mathrm{~mol} \%), \mathrm{P}^{n} \mathrm{Bu}_{3}(20 \mu \mathrm{L}, 40 \mathrm{~mol} \%), \mathrm{CuF}_{2}(6.1 \mathrm{mg}, 30 \mathrm{~mol} \%)$, base (3.0 equiv.) in solvent $(1 \mathrm{~mL}, 0.2 \mathrm{M})$ at $160^{\circ} \mathrm{C}$ for $36 \mathrm{~h}$.

${ }^{b}$ Yields given were determined by ${ }^{1} \mathrm{H}-\mathrm{NMR}$ analysis of the crude reaction mixture with 1,3,5-trimethoxybenzene.

${ }^{c}$ Yield of isolated product.

d $30 \mathrm{~mol} \% \mathrm{LiCl}$ was used as additive.

e Without $\mathrm{CuF}_{2}$.

Boc = tert-butoxycarbonyl, $C O D=1,5$-cyclooctadiene
Next, we turned our attention to the loading of $\mathrm{Ni}(\mathrm{COD})_{2}$. When the catalyst amount was reduced from 10 to $5 \mathrm{~mol} \%$, a lower yield was obtained (entry 11). Finally, different bases were tested (entries 12-14), however, better yields were not achieved. Furthermore, no product was observed in the absence of $\mathrm{CuF}_{2}$ (entry 15).

To evaluate the applicability and utility of the optimized protocol, different amide substrates were employed to determine the scope of the Ni-catalyzed deamidative silylation reaction. As illustrated in Scheme 2, different amide substrates could be converted into the corresponding aryltriethylsilanes $\mathbf{8}$ in moderate to high yields. For example, naphthyl (6a) and a wide range of phenyl $(\mathbf{6 b}-\mathbf{j})$ and heterocyclic $(\mathbf{6 k}-\mathbf{m})$ derived amides could be efficiently converted into the corresponding arylsilanes 8a-m by employing silylborane compound 7 . The protocol shows a good functional group tolerance and groups such as tert-butyl $(\mathbf{8 c})$, methyl ester (8d), methoxy (8e), methyl (8h), fluoride (8i), benzofuran (8k), benzothiophene (8I) and quinoline $(\mathbf{8 m})$ are tolerated. Para- and ortho-substitutions are also tolerated, as shown by the formation of aryl silanes $\mathbf{8 b}$ and $\mathbf{8 g}$, respectively. Notably, a high yield (95\%) was obtained for the dioxole and m-methoxy derivatives $\mathbf{8 f}$ and $\mathbf{8 j}$.

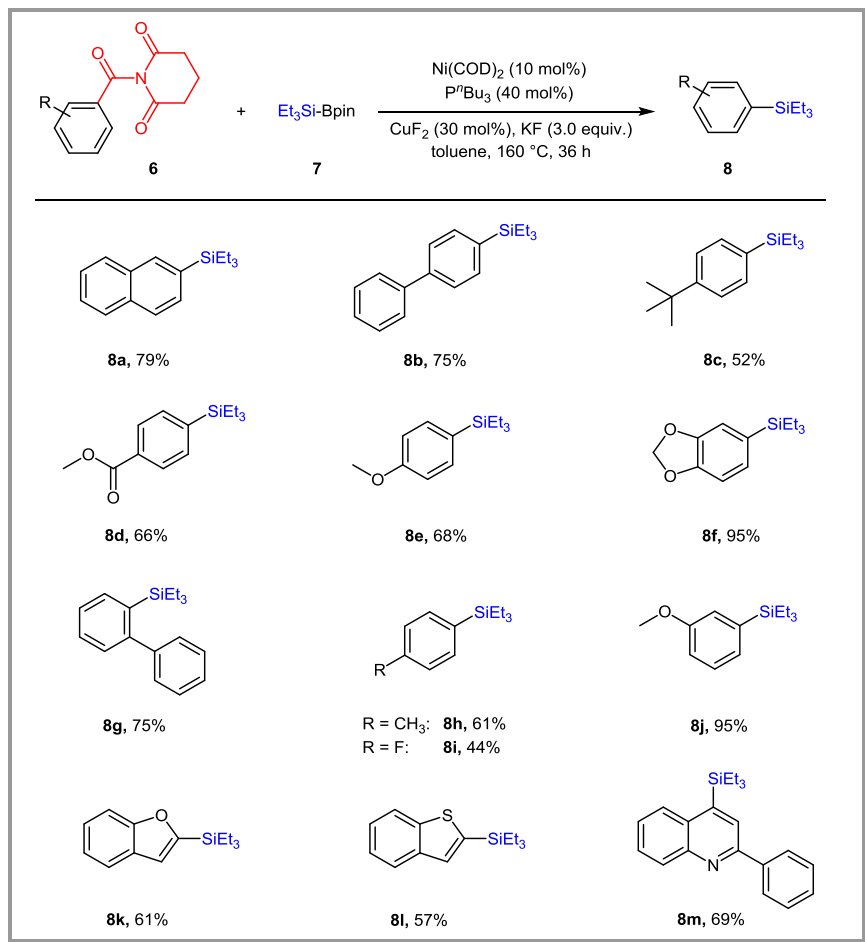

Scheme 2 Substrate scope for the silylation of aryl amides. Reagents and conditions: 6 (0.20 mmol), 7 (96.9 mg, $0.40 \mathrm{mmol}), \mathrm{Ni}(\mathrm{COD})_{2}(5.5 \mathrm{mg}, 10$ mol\%), $\mathrm{P}^{n} \mathrm{Bu}_{3}\left(20 \mu \mathrm{L}, 40\right.$ mol\%), $\mathrm{CuF}_{2}$ (6.1 mg, $\left.30 \mathrm{~mol} \%\right), \mathrm{KF}$ (34.9 mg, 3.0 equiv.), toluene $(1 \mathrm{~mL}, 0.2 \mathrm{M}), 160{ }^{\circ} \mathrm{C}, 36 \mathrm{~h}$. Yields for isolated products.

With a slightly modified protocol conversion of the amides into the corresponding arylboronate products $\mathbf{1 0}$ was also accomplished. Glutarimide substrates $\mathbf{6}$ can be easily prepared in a one-step procedure from the appropriate acyl chlorides and are therefore useful starting materials for the synthesis of arylsilanes as well as arylboronates. For the C-B bond forming protocol, ${ }^{23}$ representative substrates 6 a-f were selected to undergo reaction using bispinacolato diboron as coupling nucleophile and the products 10a-f were obtained in acceptable yields (Scheme 3). 


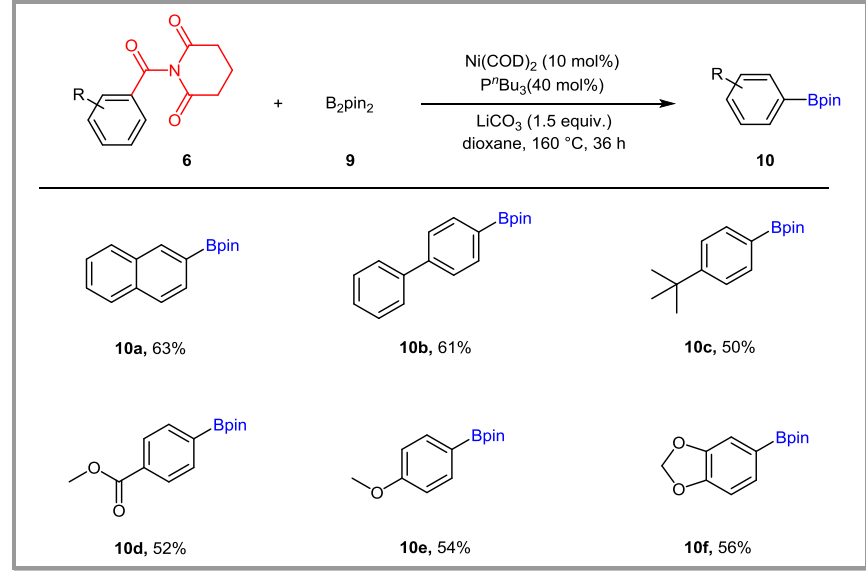

Scheme 3 Substrate scope for the borylation of aryl amides. Reagents and conditions: $6(0.20 \mathrm{mmol}), 9(76.2 \mathrm{mg}, 0.30 \mathrm{mmol}), \mathrm{Ni}(\mathrm{COD})_{2}(5.5 \mathrm{mg}, 10$ mol\%), $\mathrm{P}^{n} \mathrm{Bu}_{3}\left(20 \mu \mathrm{L}, 40\right.$ mol\%), $\mathrm{Li}_{2} \mathrm{CO}_{3}$ (22.2 mg, 1.5 equiv.), 1,4-dioxane (1 $\mathrm{mL}, 0.2 \mathrm{M}), 160{ }^{\circ} \mathrm{C}, 36 \mathrm{~h}$. Yields for isolated products. $\mathrm{B}_{2} \mathrm{pin}_{2}=$ Bis(pinacolato)diboron.

Based on the results in Schemes 3 and 4, we subsequently turned our interest to the use of imine nucleophiles to examine the decarbonylative amination of amides (Scheme 4). Based on our previous protocol, we selected benzophenone imine $\mathbf{1 1}$ as amine source with dppf as supporting ligand, $\mathrm{K}_{3} \mathrm{PO}_{4}$ as base, $\mathrm{LiCl}$ as additive, $\mathrm{Ni}(\mathrm{COD})_{2}$ as catalyst in $0.2 \mathrm{M}$ toluene at $170{ }^{\circ} \mathrm{C}$ for 24 hours under Ar. As show in Scheme 4, naphthyl (12a), parasubstituted biphenyl (12b), quinoline (12c) and dioxole (12d) derived amines were obtained in moderate yields.

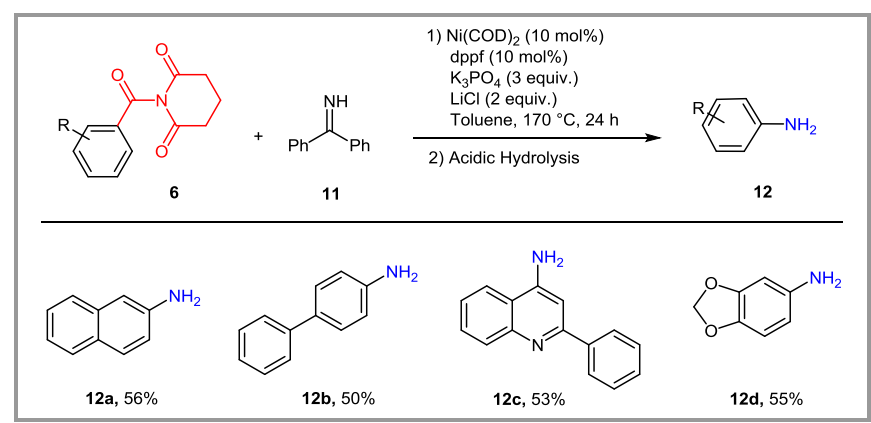

Scheme 4 Substrate scope for the amination reaction. Reagents and conditions: 6 (0.20 mmol), $11(72.5 \mathrm{mg}, 0.40 \mathrm{mmol}), \mathrm{Ni}(\mathrm{COD})_{2}(5.5 \mathrm{mg}, 10$ mol\%), dppf (11.0 mg, 10 mol\%), $\mathrm{K}_{3} \mathrm{PO}_{4}(127.0 \mathrm{mg}, 3.0$ equiv.), $\mathrm{LiCl}(17.0 \mathrm{mg}$, 2.0 equiv.), toluene $(1 \mathrm{~mL}, 0.2 \mathrm{M}), 170^{\circ} \mathrm{C}, 24 \mathrm{~h}$. Yields for isolated products.

In summary, we have developed Ni-catalyzed decarbonylative silylation, borylation and amination reactions of amides. ${ }^{24}$ Under the optimized conditions, various arylsilanes, arylboranes and arylamines, which are important building blocks in synthetic chemistry, were prepared with good yields and high stereospecificity. The here described cross coupling stands out due to the ready accessibility of substrates, mild reaction conditions and simple reaction handling allowing a stereoselective product formation. Further mechanistic studies and other related transformations are currently underway in our laboratories.

\section{Funding Information}

Click here to insert sources of funding, grant numbers, etc. Do not repeat the same in the acknowledgment.

\section{Acknowledgment}

L. Guo and H. Yue acknowledge the China Scholarship Council and H.-H. Liao the DAAD for a fellowship.

\section{Supporting Information}

YES (this text will be updated with links prior to publication)

\section{Primary Data}

Is there Primary Data to be associated with this manuscript? Click here, then the arrow, and choose YES or NO.

\section{References and Notes}

(1) (a) Modern Organonickel Chemistry Tamaru, Y. Ed.; Wiley-VCH, Weinheim, 2005; (b) Tasker ,S. Z.; Standley, E. A.; Jamison, T. F. Nature 2014, 509, 299.

(2) Wang, X.; Dai, Y.; Gong, H. Top. Curr. Chem. 2016, 374, 43.

(3) Yamaguchi, J.; Muto, K.; Itami, K. Top. Curr. Chem. 2016, 374, 55.

(4) (a) Dubbaka, S. R.; Vogel, P. Angew. Chem. Int. Ed. 2005, 44, 7674; (b) Prokopcov, H.; Kappe, C. O. Angew. Chem. Int. Ed. 2009, 48, 2276; (c) Wang, L.; Hea, W.; Yu, Z. Chem. Soc. Rev. 2013, 42, 599; (d) Pana, F.; Shi, Z.-J. ACS Catal. 2014, 4, 280.

(5) (a) Rosen, B. M.; Quasdorf, K. W.; Wilson, D. A.; Zhang, N.; Resmerita, A.-M.; Garg, N. K.; Percec, V. Chem. Rev. 2010, 111, 1346; (b) Yu, D.-G.; Li, B.-J.; Shi, Z.-J. Acc. Chem. Res. 2010, 43, 1486; (c) McGlacken, G. P.; Clarke, S. L. ChemCatChem 2011, 3, 1260; (d) Mesganaw, T.; Garg, N. K. Org. Process Res. Dev. 2013, 17, 29; (e) Kozhushkov, S. I.; Potukuchiw, H. K.; Ackermann, L. Catal. Sci. Technol. 2013, 3, 562; (f) Cornella, J.; Zarate, C.; Martin, R. Chem. Soc. Rev. 2014, 43, 8081; (g) Tobisu, M.; Chatani, N. Top. Curr. Chem. 2016, 374, 41.

(6) Wang, Q.; Su, Y.; Li, L.; Huang, H. Chem.Soc.Rev. 2016, 45, 1257.

(7) (a) Dzik, W. I.; Lange, P. P.; Gooßen, L. J. Chem. Sci. 2012, 3, 2671. (b) Yamaguchi, J.; Itami, K. in Metal-Catalyzed Cross-Coupling Reactions and More; Vol 3, de Meijere, A.; Bräse, S.; Oestreich, M., Eds.; Wiley-VCH Weinheim, 2014, pp. 1353. (c) New trends in cross-coupling: Theory and applications, Colacot, T. J., Ed.; RSC: Cambridge, UK, 2015.

(8) Examples of decarbonylative cross couplings of acyl chlorides: (a) Blaser, H.-U.; Spencer, A. J. Organomet. Chem. 1982, 233, 267; (b) Obora, Y.; Tsuji, Y.; Kawamura, T. J. Am. Chem. Soc. 1993, 115, 10414; (c) Zhao, X.; Yu, Z. J. Am. Chem. Soc. 2008, 130, 8136; (d) Ye, W.; Luo, N.; Yu, Z. Organometallics 2010, 29, 1049.

(9) Decarbonylative cross couplings of carboxylic anhydrides: (a) O'Brien, E. M.; Bercot, E. A.; Rovis, T. J. Am. Chem. Soc. 2003, 125, 10498; (b) Gooßen, L. J.; Paetzold, J. Adv. Synth. Catal. 2004, 346, 1665; (c) Kajita, Y.; Kurahashi, T.; Matsubara, S. J. Am. Chem. Soc. 2008, 130, 17226; (d) Jin, W.; Yu, Z.; He, W.; Ye, W.; Xiao, W.-J. Org. Lett. 2009, 11, 1317; (e) Prakash, R.; Shekarrao, K.; Gogoi, S.; Boruah, R. C. Chem. Commun. 2015, 51, 9972.

(10) (a) Gooßen, L. J.; Paetzold, J. Angew. Chem. Int. Ed. 2002, 41, 1237; (b) Gooßen, L. J.; Paetzold, J. Angew. Chem. Int. Ed. 2004, 43, 1095; (c) Gribkov, D. V.; Pastine, S. J.; Schnürch, M.; Sames, D. J. Am. Chem. Soc. 2007, 129, 11750; (d) Okita, T.; Kumazawa, K.; Takise, R.; Muto, K.; Itami, K.; Yamaguchi, J. Chem. Lett. 2017, 46, 218. For examples of $\mathrm{Ni}$-catalyzed decarbonylative transformations of esters for C-C bond formations, see: (e) Amaike, K.; Muto, K.; Yamaguchi, J.; Itami, K. J. Am. Chem. Soc. 2012, 134, 13573; (f) Correa, A.; Cornella J.; Martin, R. Angew. Chem. Int. Ed. 2013, 52, 1878; (g) Meng, L.; Kamada, Y.; Muto, K.; Yamaguchi, J.; Itami, K. Angew. Chem., Int. Ed. 2013, 52, 10048; (h) Hong, X.; Liang, Y.; Houk, K. N. J. Am. Chem. Soc. 2014, 136, 2017; (i) Lu, Q.; Yu, H.; Fu, Y. J. Am. Chem. Soc. 2014, 136, 8252; (j) Muto, K.; Yamaguchi, J.; Musaev, D. G.; Itami, K.; Nat. Commun. 2015, 6, 7508; (k) Desnoyer, A. N.; Friese, F. W.; Chiu, W.; Drover, M. W.; Patrick, B. O.; Love, J. A. Chem. Eur. J. 2016, 22, 4070; (l) Amaike, K.; Itami, K.; Yamaguchi, J. Chem.-Eur. J. 2016, 22, 4384; (m) Takise, R.; Isshiki, R.; Muto, K.; Itami, K.; Yamaguchi, J. J. Am. Chem. Soc. 2017, 139, 3340; (n) Liu, X.; Jia, J.; Rueping, M. ACS Catal. 2017, 7, 4491. For 
borylation and silylation, see: (o) Guo, L.; Chatupheeraphat, A.; Rueping, M. Angew. Chem. Int. Ed. 2016, 55, 11810; (p) Pu, X.; Hu, J.; Zhao, Y.; Shi, Z. ACS Catal. 2016, 6, 6692; (q) Guo, L.; Rueping, M. Chem. Eur. J. 2016, 22, 16787. For amination, see: (r) Yue, H.; Guo, L.; Liao, H.-H.; Cai, Y.; Zhu, C.; Rueping, M. Angew. Chem. Int. Ed. 2017, 56, 4282 .

(11) For reviews, see: (a) Meng, G.; Shi, S.; Szostak, M. Synlett 2016, 27, 2530; (b) Dander, J. E.; Garg, N. K. ACS Cat. 2017, 7, 1413; (c) Liu, C.; Szostak, M. Chem. Eur. J. 2017, 23, 7157.

(12) For examples of Ni-catalyzed decarbonylative transformations of amides for C-C bond formations, see: (a) Shi, S.; Meng, G.; Szostak, M. Angew. Chem., Int. Ed. 2016, 55, 6959. (b) Srimontree, W.; Chatupheeraphat, A.; Liao, H.-H.; Rueping, M. Org. Lett. 2017, 19, 3091. For borylation, amination and reduction, see: (c) Hu, J.; Zhao, Y.; Liu, J.; Zhang, Y.; Shi, Z. Angew. Chem., Int. Ed. 2016, 55, 8718. (d) Dey, A.; Sasmal, S.; Seth, K.; Lahiri, G. K.; Maiti, D. ACS Catal. 2017, 7, 433. (e) Yue, H.; Guo, L.; Lee, S.-C.; Liu, X.; Rueping, M. Angew. Chem. Int. Ed. 2017, 56, 3972. (f) Hu, J.; Wang, M.; Pu, X.; Shi, Z. Nat. Commun. 2017, 8, 14993. (g) B. J. Simmons, M. Hoffmann, J. Hwang, M. K. Jackl, N. K. Garg, Org. Lett. 2017, 19, 1910. For examples of $\mathrm{Pd}$ and Rh-catalyzed decarbonylative transformations of amides, see: (h) Meng, G.; Szostak, M. Angew. Chem., Int. Ed. 2015, 54, 14518. (i) Liu, C.; Meng, G.; Szostak, M. J. Org. Chem. 2016, 81, 12023. (j) Meng, G.; Szostak, M. Org. Lett. 2016, 18, 796. (k) Wu, H.; Liu, T.; Cui, M.; Li, Y.; Jian, J.; Wang, H.; Zeng, Z. Org. Biomol. Chem. 2017, 15, 536. (l) Shi, S.; Szostak, M. Org. Lett. 2017, 19, 3095.

(13) (a) Leiendecker, M.; Hsiao, C. C.; Guo, L.; Alandini, N.; Rueping, M. Angew. Chem. Int. Ed. 2014, 53, 12912. (b) Guo, L.; Leiendecker, M.; Hsiao, C.-C.; Baumann, C.; Rueping, M. Chem. Commun. 2015, 51, 1937. (c) Leiendecker, M.; Chatupheeraphat, A.; Rueping, M. Org. Chem. Front. 2015, 2, 350. (d) Liu, X.; Hsiao C.-C.; Kalvet, I.; Leiendecker, M.; Guo, L.; Schoenebeck, F.; Rueping, M. Angew. Chem. Int. Ed. 2016, 55, 6093. (e) Guo, L.; Hsiao, C.-C.; Yue, H.; Liu, X.; Rueping, M. ACS Catal. 2016, 6, 4438-4442. (f) Guo, L.; Liu, X.; Baumann, C.; Rueping, M. Angew. Chem. Int. Ed. 2016, 55, 15415. (g) Fan, L.; Jia, J.; Hou, H.; Lefebvre, Q.; Rueping, M. Chem. Eur. J. 2016, 22, 16437. (h) Yue, H.; Guo, L.; Liu, X.; Rueping, M. Org. Lett. 2017, 19, 1788.

(14) For reviews on organosilicon compounds, see: (a) Corey, J. Y.; Braddock-Wilking, J. Chem. Rev. 1999, 99, 175; (b) Brook, M. Silicon in Organic, Organometallic and Polymer Chemistry, Wiley, New York, 2000; (c) Denmark, S. E.; Sweis, R. F. Acc. Chem. Res. 2002, 35, 835; (d) Marciniec, B. Coord. Chem. Rev. 2005, 249, 2374; (e) Nakao, Y.; Hiyama, T. Chem. Soc. Rev. 2011, 40, 4893; (f) Cheng, C.; Hartwig, J. F. Chem. Rev. 2015, 115, 8946.

(15) For reviews on organoboron compounds, see: (a) Miyaura, N.; Suzuki, A. Chem. Rev. 1995, 95, 2457; (b) Miyaura, N. Top. Curr. Chem. 2002, 219, 11; (c) Miyaura, N. Bull. Chem. Soc. Jpn. 2008, 81, 1535.

(16) (a) Showwell, G. A.; Mills, J. S. Drug Discov. Today 2003, 8, 551; (b) Denmark, S. E.; Liu, J. H.-C. Angew. Chem. Int. Ed. 2010, 49, 2978; (c) Franz, A. K.; Wilson, S. O. J. Med. Chem. 2013, 56, 388.

(17) Hall, D. G. Boronic Acids: Preparation and Applications in Organic Synthesis and Medicine, Wiley-VCH: Weinheim, 2005.

(18) (a) Ricci, A., Ed. Amino Group Chemistry: From Synthesis to the Life Sciences; Wiley-VCH: Weinheim, 2008; (b) Lawerence, S. A. Amines: Synthesis, Properties and Applications, Cambridge University, Cambridge, 2004; (c) Brown, B. R. The Organic Chemistry of Aliphatic Nitrogen Compounds, Cambridge University, Cambridge, 2004; (d) Rappoport, Z., Ed. The Chemistry of Anilines, Parts 1 and 2; John Wiley \& Sons: New York, 2007.
(19) (a) Manoso, A. S.; Ahn, C.; Soheili, A.; Handy, C. J.; Correia, R.; Seganish, W. M.; DeShong, P. J. Org. Chem. 2004, 69, 8305; (b) Pintaric, C.; Olivero, S.; Gimbert, Y.; Chavant, P. Y.; Duñach, E. J. Am. Chem. Soc. 2010, 132, 11825.

(20) For a review on the silylation of organic halides, see: (a) Murata, M.; Masuda, Y. J. Synth. Org Chem. 2010, 68, 845; For recent reviews on the borylation of organic halides, see: (b) Kubota, K.; Iwamoto, H.; Ito, H. Org. Biomol. Chem. 2017, 15, 285; (c) Murata, M. Heterocycles 2012, 85, 1795; For a review on silylation and borylation of organic halides, see: (d) Murata, M. in Science of Synthesis (Eds. Molander, G. A.; Wolfe, J. P.; Larhed, M) 2013, 2, 439.

(21) For recent reviews on C-H bond silylation, see: (a) Cheng, V.; Hartwig, J. F. Chem. Rev. 2015, 115, 8946; (b) Yang, Y.; Wang, C. Sci. China Chem. 2015, 58, 1266; (c) Sharma, R.; Kumar, R.; Kumar, I.; Singh, B.; Sharma, U. Synthesis 2015, 47, 2347. (d) Xu, Z.; Huang, W.-S.; Zhang, J.; Xu, L.-W. Synthesis 2015, 47, 3645. (e) Xu, Z.; Xu, L.-W. ChemSusChem 2015, 8, 2176. For recent reviews on C-H bond borylation, see: (f) Mkhalid, I. A. I.; Barnard, J. H.; Marder, T. B.; Murphy, J. M.; Hartwig, J. F. Chem. Rev. 2010, 110, 890; (g) Hartwig, J. F. Chem. Soc. Rev. 2011, 40, 1992; (h) Ros, A.; Fernandez, R.; Lassaletta, J. M. Chem. Soc. Rev. 2014, 43, 3229.

(22) (a) Zarate, C.; Martin, R. J. Am. Chem. Soc. 2014, 136, 2236; For the $\mathrm{Ni}$ catalyzed silylation of aryl, methyl ethers, see: (b) Zarate, C.; Nakajima, M.; Martin, R. J. Am. Chem. Soc. 2017, 139, 1191. For the Ni catalyzed silylation of silyloxyarenes, see: Wiensch, E. M.; Todd, D. P.; Montgomery, J. ACS Catal. 2017, 7, 5568.

(23) Recently, a protocol for the decarbonylative borylation of amides was established by Shi and co-workers, see reference 12c. With a catalytic system consisting of nickel and an $\mathrm{N}$-heterocyclic carbene ligand, the -N-Boc-N-Me derived amides were converted into the corresponding borylation products in moderate to high yields. Interestingly, distorted cyclic imides were completely unsuccessful.

(24) General Procedure for the Nickel-catalyzed decarbonylative silylation of arylamides via a deamidative reaction pathway. In a nitrogen-filled glovebox, a $10-\mathrm{mL}$ oven-dried sealed tube containing a stirring bar was charged with the corresponding amide ( $0.20 \mathrm{mmol}$, 1.0 equiv), yellow $\mathrm{Ni}(\mathrm{COD})_{2}$ ( $5.5 \mathrm{mg}, 10 \mathrm{~mol} \%$ ), copper fluoride (II) $(6.1 \mathrm{mg}, 30 \mathrm{~mol} \%)$ and potassium fluoride (34.9 mg, $0.60 \mathrm{mmol}, 3.0$ equiv). Subsequently, freshly distilled toluene $(1.0 \mathrm{~mL})$ was added, and then triethylsilylborane (96.9 $\mathrm{mg}, 0.40 \mathrm{mmol}, 2.0$ equiv) and tri- $n$-butylphosphine ligand $(20 \mu \mathrm{L}$, $40 \mathrm{~mol} \%$ ) were added respectively via microsyringe. The tube with the mixture was sealed and removed from the glovebox. After stirring at $160{ }^{\circ} \mathrm{C}$ for $36 \mathrm{~h}$, the mixture was allowed to cool to room temperature, diluted with EtOAc $(5 \mathrm{~mL})$ and filtered through a celite plug, eluting with additional EtOAc $(10 \mathrm{~mL})$. The filtrate was concentrated and purified by column chromatography on silica gel to yield the product.

Synthesis of 8i: As for general procedure I, starting from 1-(4fluorobenzoyl)piperidine-2,6-dione $(47.0 \mathrm{mg}, 0.20 \mathrm{mmol})$, the title product was isolated as colorless oil after flash chromatography on silica gel, $18.5 \mathrm{mg}(44 \%) .{ }^{1} \mathrm{H}$ NMR $(600 \mathrm{MHz}$, $\left.\mathrm{CDCl}_{3}\right): \delta=7.48-7.43(\mathrm{~m}, 2 \mathrm{H}), 7.08-7.01(\mathrm{~m}, 2 \mathrm{H}), 0.95(\mathrm{t}, J=7.8 \mathrm{~Hz}$, $9 \mathrm{H}), 0.8-0.76(\mathrm{~m}, 6 \mathrm{H}) ;{ }^{13} \mathrm{C} \mathrm{NMR}\left(150 \mathrm{MHz}, \mathrm{CDCl}_{3}\right): \delta=164.3,162.7$ $136.0,135.9,132.7,132.7,114.9,114.7,7.3,3.4 ;{ }^{19} \mathrm{~F}$ NMR (564 $\mathrm{MHz}, \mathrm{CDCl}_{3}$ ): $\delta=-112.69$; IR (ATR): $\tilde{v}=3031,2951,2882,2327$, 2102, 1898, 1586, 1498, 1459, 1231, 1161, 1100, 1007, 818, 721 $\mathrm{cm}^{-1}$; MS (ESI): $\mathrm{m} / \mathrm{z}(\%)=210.0\left(\mathrm{M}^{+}, 38\right), 182.1(28), 181.0(47)$, $153.0(20)$. 